\title{
Magnetic Mott criticality in a $\kappa$-type organic salt probed by NMR
}

\author{
Fumitaka Kagawa ${ }^{\star \dagger}$, Kazuya Miyagawa and Kazushi Kanoda
}

\begin{abstract}
Near a Mott transition', which can be tuned by controlling either the charge-carrier density ('filling') or the correlation strength ('bandwidth'), lies fascinating emergent behaviour, such as unconventional superconductivity ${ }^{2,3}$, and the understanding of the underlying Mott criticality is a longstanding challenge. Recent studies have showed that the bandwidthcontrolled Mott criticality (BCMC) involves critical fluctuations in charge $e^{4,5}$ and lattice ${ }^{6,7}$ degrees of freedom. Spin is another degree of freedom and its antiferromagnetic fluctuations are ubiquitous in strongly correlated electrons ${ }^{8,9}$. However, the magnetic aspects of BCMC are unexplored. Here, we report on the magnetic criticality brought about by BCMC. Through NMR investigations on a $\kappa$-type organic salt, we observe critical suppression of antiferromagnetic fluctuations accompanied by the critical enhancement of conductance. The two criticalities show the same exponent within experimental error. Site-to-site electron hopping introduces doubly occupied and empty sites, which extinguish stroboscopically the local spins, probably resulting in the identical criticality in charge and spin.
\end{abstract}

The investigation of BCMC has been a challenging issue, for the following two reasons. First, in many materials, BCMC is obscured by simultaneous symmetry breakings in spin or orbital degrees of freedom. Second, an atypical experimental technique, that is, continuous control of the bandwidth at a given temperature, is required. Organic compounds ${ }^{10}$ of the type $\kappa$-(BEDT-TTF $)_{2} \mathrm{X}$ are one of few examples showing the 'pure' bandwidth-controlled Mott transition without symmetry breaking, where BEDT-TTF denotes bis(ethylenedithio)tetrathiafulvalene and $\mathrm{X}$ denotes an anion. The compound investigated here, $\kappa$-(BEDT-TTF $)_{2} \mathrm{Cu}\left[\mathrm{N}(\mathrm{CN})_{2}\right] \mathrm{Cl}$ (hereafter abbreviated to $\kappa-\mathrm{Cl}$ ), is a half-filled quasi-two-dimensional system ${ }^{11}$ composed of the conducting BEDT-TTF dimer layer and an insulating anion $\mathrm{X}$ layer (Fig. 1a). Its pressure-temperature $(P-T)$ phase diagram (Fig. 1b $)^{12}$ possesses the characteristics of a pure bandwidth-controlled Mott transition: the first-order Mott transition line free from magnetic symmetry breaking terminates at a critical end-point $t^{5,6,12-15}$. Theoretically, the global feature of this phase diagram is now well reproduced by the cluster dynamical mean field theory ${ }^{16,17}$ (DMFT). Note that the pressure-induced Mott transition at the end-point $\left(T_{\mathrm{c}}\right)$ is of second order; thus, BCMC is expected to grow towards the finite-temperature end-point in a divergent manner ${ }^{18,19}$ without being cut off by the discontinuous first-order transition.

According to DMFT (ref. 20), the continuous bandwidthcontrolled Mott transition at the end-point is accompanied by the critical behaviour in various quantities ${ }^{18,21}$, such as the density of doubly occupied sites (doublons), the height of the quasiparticle peak in photoemission and conductance: among them, the critical behaviour of conductance has been experimentally confirmed in
$\left(\mathrm{V}_{1-x} \mathrm{Cr}_{x}\right)_{2} \mathrm{O}_{3}$ (ref. 4) and $\kappa-\mathrm{Cl}$ (ref. 5). However, the effects on the magnetic properties are yet to be investigated experimentally and theoretically. To address the magnetic aspect of BCMC, we used ${ }^{13} \mathrm{C}$-substituted $\kappa$-Cl (abbreviated to ${ }^{13} \mathrm{C}-\kappa-\mathrm{Cl}$ ) and carried out ${ }^{13} \mathrm{C}$-NMR measurements (see the Methods section), which is a powerful probe of local spin dynamics. For the bandwidth control, NMR was combined with a continuously controllable pressure technique, which is an effective method for this highly compressible organic salt. Below, we focus on the NMR properties around the end-point and present three regimes of bandwidth-controlled Mott transition: discontinuous Mott transition (below the end-point temperature, $T_{\mathrm{c}}$ ), insulator-bad metal crossover (above $T_{\mathrm{c}}$ ) and continuous Mott transition at $T_{\mathrm{c}}$.

Figure $2 \mathrm{a}$ shows the pressure dependence of ${ }^{13} \mathrm{C}$-NMR spectra at $31.6 \mathrm{~K}$ (below $T_{\mathrm{c}}$ ). It is seen that the two broad lines observed at low pressures change discontinuously around $20 \mathrm{MPa}$ into the sharp two-line spectra at high pressures through their coexistence, which is also observed under chemical pressure control ${ }^{22}$. Referring to the $P-T$ phase diagram (Fig. 1b), it is obvious that the former is from the insulating phase, whereas the latter is from the bad metallic one. The volume fraction of the bad metallic phase shows a steep change, consistent with the first-order Mott transition (Fig. 2b). Reasonably, the pressure dependence of $1 / T_{1} T$, which is a measure of antiferromagnetic fluctuations (see the Methods section), is also discontinuous (Fig. 2c), and the clear critical phenomena of $1 / T_{1} T$ are absent at this temperature. In the insulating phase, as pressure increases, both the linewidth (Fig. 2a) and $1 / T_{1} T$ decrease, indicating the suppression of antiferromagnetic fluctuations. Note that this change is accompanied by a suppression of the Mott gap $^{13,14,23}$ and an increase in conductance $G$ (Fig. 2d), although the conductance in the insulating phase is still much smaller than that in the bad metallic phase. These facts suggest that the tiny increase in doublons in the insulating phase (doublonpoor state) markedly reduces antiferromagnetic fluctuations. In contrast, in the bad metallic phase (doublon-rich state), the linewidth and $1 / T_{1} T$ are nearly pressure independent in the present range. A sudden drop in $1 / T_{1} T$ from the values in the insulating phase implies that antiferromagnetic fluctuations are relatively suppressed.

In the insulator-bad-metal crossover regime above $T_{\mathrm{c}}$ ( $\approx 40-43 \mathrm{~K}$, see below), the NMR spectra keep the two-line nature, as seen in the data at $43.4 \mathrm{~K}$ (Fig. 3a). This indicates the absence of a first-order transition. Correspondingly, the pressure dependence of $1 / T_{1} T$ (Fig. $3 \mathrm{~b}$ ) is continuous and decreases monotonously; nevertheless, the $1 / T_{1}(P) T$ curve shows inflexion at the pressure close to the transport crossover pressure ${ }^{5}$ (Fig. 3c). This coincidence again suggests that the increase in doublons leads to the suppression of antiferromagnetic fluctuations. 
a

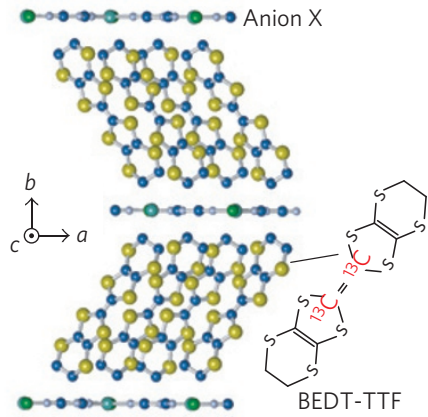

b

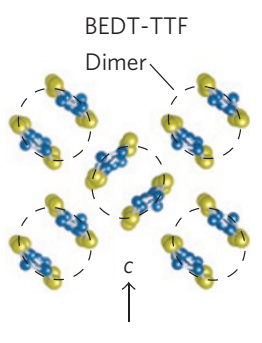

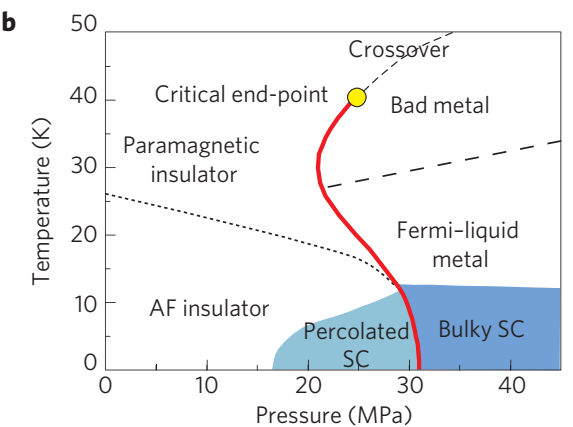

Figure 1 | Structure of $\kappa$-(BEDT-TTF $)_{2} \mathbf{C u}\left[\mathbf{N}(\mathbf{C N})_{2}\right] \mathbf{C l}$ and the bandwidth-controlled Mott transition. a, Crystal structure of $\kappa$-(BEDT-TTF) ${ }_{2} \mathrm{Cu}$ $\left[\mathrm{N}(\mathrm{CN})_{2}\right] \mathrm{Cl}$. In the two-dimensional conducting layers (the ac plane), BEDT-TTF molecules form dimers. The dimer has one hole and thus the band is half-filled. The two central carbon atoms of BEDT-TTF are labelled with ${ }^{13} \mathrm{C}$ isotopes for NMR measurements. $\mathbf{b}$, Pressure-temperature phase diagram of $\kappa$-(BEDT-TTF) ${ }_{2} \mathrm{Cu}\left[\mathrm{N}(\mathrm{CN})_{2}\right] \mathrm{Cl}$ taken from refs 5, 6 and 12-15. The bold line represents the first-order Mott transition. 'AF' and 'SC' denote antiferromagnetic and superconductor, respectively.

a

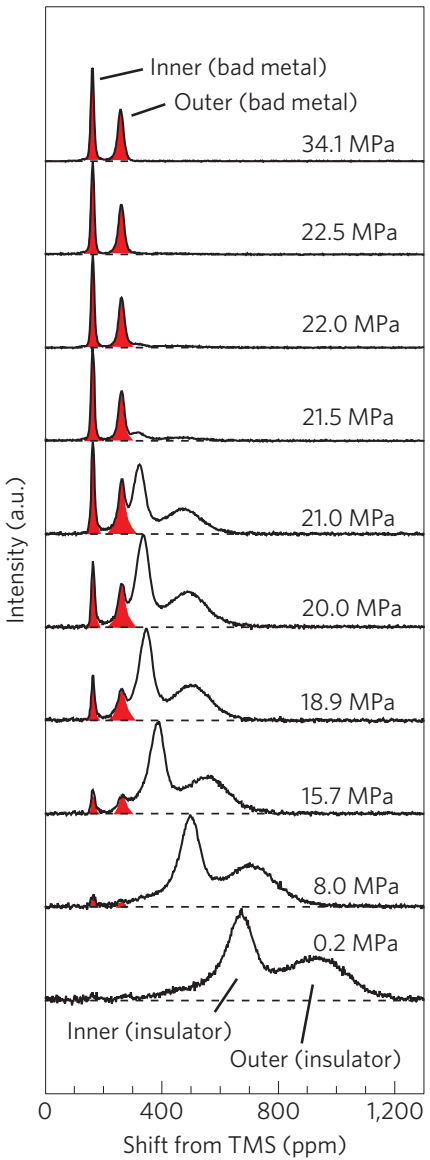

b

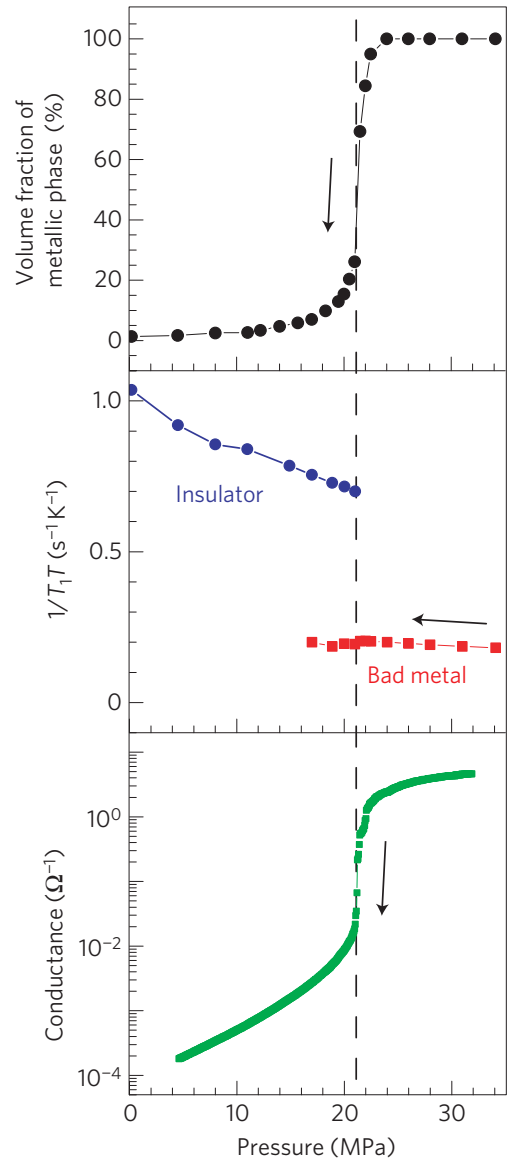

Figure 2 | Bandwidth-controlled discontinuous Mott transition seen from ${ }^{13} \mathbf{C}-\mathbf{N M R}$. a-c, Pressure dependence of ${ }^{13} \mathrm{C}-\mathrm{NMR}$ spectra (a), volume fraction of bad metallic phase estimated from the spectral weight $(\mathbf{b})$ and $1 / T_{1} T$ of the outer site $(\mathbf{c})$ at $31.6 \mathrm{~K}\left(<T_{\mathrm{c}}\right)$. In $\mathbf{a}$, the spectra coming from the bad metallic phase are shaded red as a guide to the eye. TMS: tetramethylsilane. $\mathbf{d}$, The conductance data for ${ }^{12} \mathrm{C}-\kappa-\mathrm{Cl}$ (that is, ${ }^{13} \mathrm{C}$-non-substituted $\kappa$ - $\mathrm{Cl}$ ) at $32.1 \mathrm{~K}$ (from ref. 14), for comparison. All of the data were taken in a decreasing pressure process. The pressure hysteresis is expected to be $\sim 0.2 \mathrm{MPa}$ (ref. 5).

Next, we focus on how BCMC (for example, the criticality of conductance) at the continuous Mott transition affects $1 / T_{1} T$. For this purpose, NMR measurements have to be done at a temperature very close to $T_{\mathrm{c}}$. In the NMR spectra of ${ }^{13} \mathrm{C}-\kappa-\mathrm{Cl}$ at $39.7 \mathrm{~K}$, for which the $T_{\mathrm{c}}$ is determined from previous conductance measurements ${ }^{5}$ for ${ }^{13} \mathrm{C}$-non-substituted $\kappa-\mathrm{Cl}$ (abbreviated to ${ }^{12} \mathrm{C}-\kappa-\mathrm{Cl}$ ), we found insulator-bad metal coexistence and an abrupt change in shift, which are indications of a weak first-order transition (see Supplementary Fig. S1). Therefore, $T_{\mathrm{c}}$ of ${ }^{13} \mathrm{C}-\kappa-\mathrm{Cl}$ is probably located at $40-43 \mathrm{~K}$ and we regard $41.5 \mathrm{~K}$ as very close to $T_{\mathrm{c}}$. This slight difference in $T_{\mathrm{c}}$ is attributable to the ${ }^{13} \mathrm{C}$-substitution effect ${ }^{24}$; in fact, in deuterated $\kappa$-(BEDT-TTF $)_{2} \mathrm{Cu}\left[\mathrm{N}(\mathrm{CN})_{2}\right] \mathrm{Br}, T_{\mathrm{c}}$ is found to rise by a few kelvin as a result of the ${ }^{13} \mathrm{C}$-substitution ${ }^{24}$.

The NMR spectra also keep the two-line nature all the way across the nearly continuous Mott transition (at $41.5 \mathrm{~K}$ ); however, unlike the case of the insulator-bad metal crossover, the $1 / T_{1}(P) T$ curve shows a steep change around the critical pressure $P_{\mathrm{c}}(\approx 25.8 \mathrm{MPa})$ with a nearly vertical gradient (Fig. $\left.4 \mathrm{a}\right)$. 
a

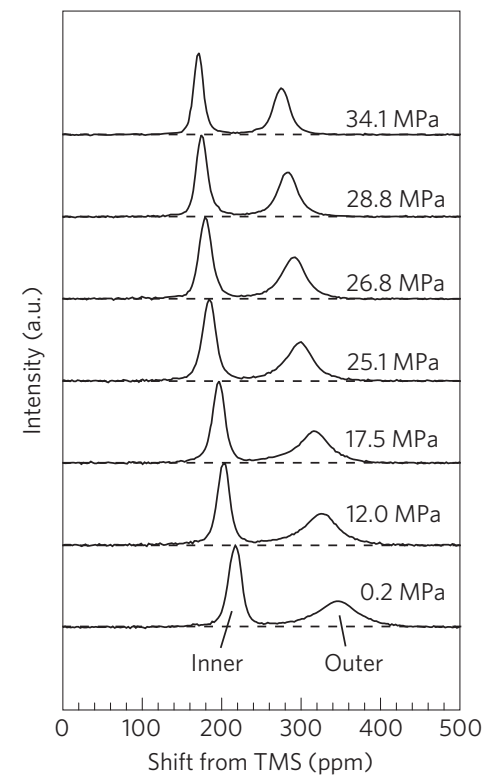

b

C

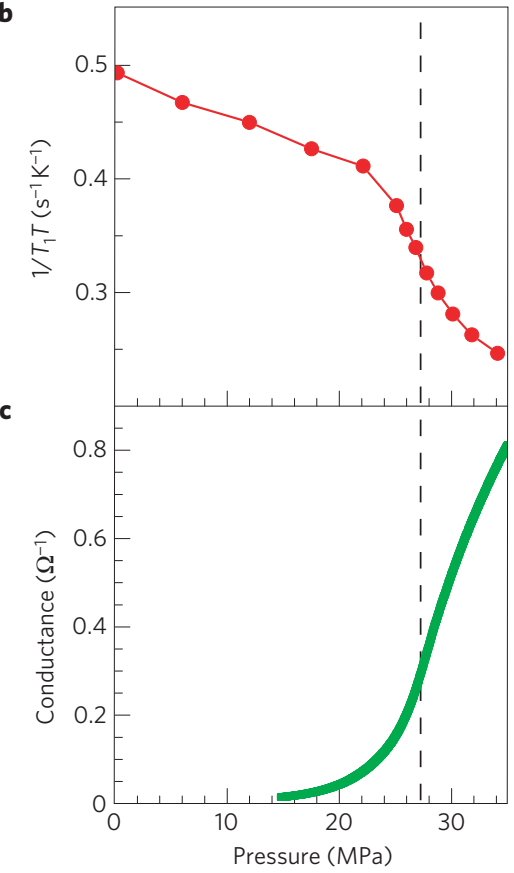

Figure 3 | Insulator-bad metal crossover seen from ${ }^{13} \mathbf{C}-\mathbf{N M R}$. a,b, Pressure dependence of ${ }^{13} \mathrm{C}-\mathrm{NMR}$ spectra $(\mathbf{a})$ and $1 / T_{1} T$ of the outer site $(\mathbf{b})$ at $43.4 \mathrm{~K}$ $\left(>T_{\mathrm{c}}\right) . \mathbf{c}$, The pressure dependence of conductance in ${ }^{12} \mathrm{C}-\kappa-\mathrm{Cl}$ (that is, ${ }^{13} \mathrm{C}$-non-substituted $\kappa-\mathrm{Cl}$ ) at $43.2 \mathrm{~K}$ (from ref. 5), for comparison.

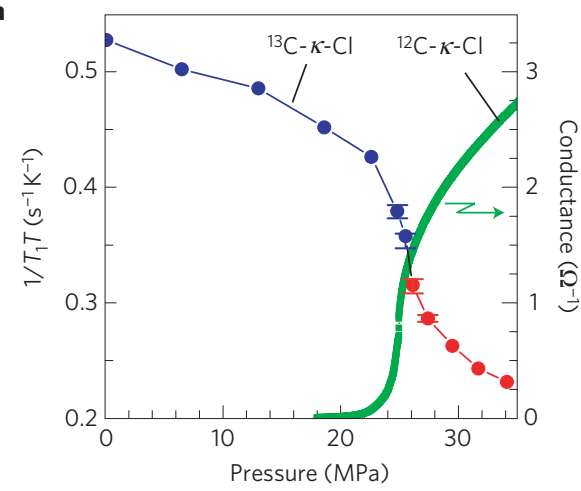

b

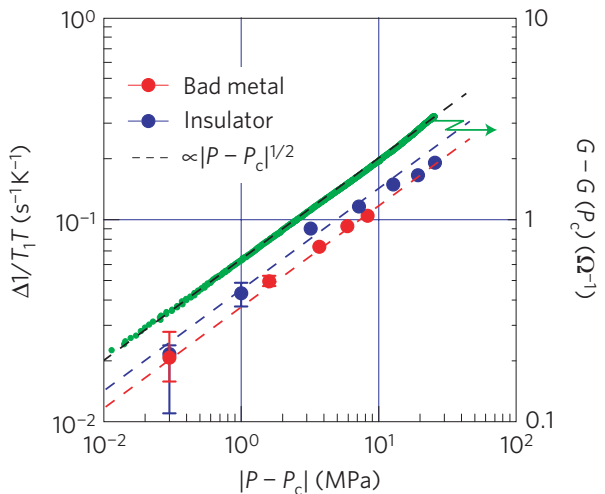

Figure 4 | Continuous Mott transition at the critical temperature seen from ${ }^{\mathbf{1 3}} \mathbf{C}$-NMR and conductance. a, Pressure dependence of $1 / T_{1} T$ of the outer site and conductance. $1 / T_{1} T$ values are the data for ${ }^{13} \mathrm{C}-\kappa-\mathrm{Cl}$ at $41.5 \mathrm{~K}$ (close to $T_{\mathrm{c}}$ of ${ }^{13} \mathrm{C}-\kappa-\mathrm{Cl}$ ), whereas the conductance (represented from ref. 5) is for ${ }^{12} \mathrm{C}-\kappa-\mathrm{Cl}$ at $39.7 \mathrm{~K}$ (close to $T_{\mathrm{c}}$ of $\left.{ }^{12} \mathrm{C}-\kappa-\mathrm{Cl}\right)$. b , Logarithmic plot of $1 / T_{1} T$ and conductance measured from the end-point against $\left|P-P_{\mathrm{c}}\right|$. The dashed black line indicates the pressure dependence of $\sim\left|P-P_{\mathrm{c}}\right|^{1 / 2}$. Regarding the plot of conductance, the data above $P_{\mathrm{c}}$ are used. The error bars show the distribution of shift-dependent $1 / T_{1}$.

Note that the $G(P)$ curve of ${ }^{12} \mathrm{C}-\kappa-\mathrm{Cl}$ at $39.7 \mathrm{~K}$ also shows a critical change at the end-point ${ }^{5}$ (that is, the critical behaviour of conductance, see Fig. $4 \mathrm{a}$ ). This qualitative similarity leads us to conclude that BCMC is emergent not only in the conductance but also in the magnetic property as a critical change of $1 / T_{1} T$ (that is, antiferromagnetic fluctuations). Remarkably, the similarity between $G(P)$ and $1 / T_{1}(P) T$ is quantitative as illustrated in Fig. $4 \mathrm{~b}$, which shows $\Delta G\left[\equiv G(P)-G\left(P_{\mathrm{c}}\right)\right]$ of ${ }^{12} \mathrm{C}-\kappa-\mathrm{Cl}$ above $P_{\mathrm{c}}$ and $\Delta 1 / T_{1} T\left[\equiv\left|1 / T_{1}(P) T-1 / T_{1}\left(P_{\mathrm{c}}\right) T\right|\right]$ of ${ }^{13} \mathrm{C}-\kappa-\mathrm{Cl}$ against $\left|P-P_{\mathrm{c}}\right|$. From the simplest linear fitting in the logarithmic scales, it is found that $\Delta 1 / T_{1} T$ obeys roughly $\left|P-P_{\mathrm{c}}\right|^{1 / \delta}$ with a critical exponent $\delta \approx 2$ in both the insulating and bad metallic phases; here note that $\delta \approx 2$ was originally found for $\Delta G$ (ref. 5 and see also Fig. 4 b). Although the relationship between $1 / T_{1} T$ and conductance is not theoretically explored at present, the agreement of $\delta$ implies that there is a linear coupling between $\Delta 1 / T_{1} T$ and $\Delta G$. Hence, if $\Delta G$ reflects the order parameter of the bandwidth-controlled Mott transition as argued in DMFT (refs 4, 21) (the relationship may be non-trivial ${ }^{25}$ ), it follows that $\Delta 1 / T_{1} T$ reflects the order parameter as well. Therefore, within this assumption, Fig. $4 \mathrm{~b}$ supports the unconventional exponent $\delta=2$. Although the unconventional $\delta$ is inconsistent with the DMFT result, that is, Ising universality class ${ }^{18}$, this value may be relevant to the quantum criticality of the Mott transition discussed in ref. 19.

BCMC seen from $1 / T_{1} T$ is summarized in Fig. 5; a clear jump emergent at low temperatures vanishes around $T_{c}(\approx 40-43 \mathrm{~K})$; and the crossover behaviour above $T_{\mathrm{c}}$ becomes moderate at higher temperatures. This temperature and pressure profile of $1 / T_{1} T$ and the NMR shift (see Supplementary Fig. S2), which could not be captured in the previous ${ }^{1} \mathrm{H}-\mathrm{NMR}$ measurement ${ }^{12}$, are in common with those of conductance ${ }^{5}$. In Fig. 5, 1/ $T_{1} T$ has no peak structure around the end-point, implying that both the divergence of antiferromagnetic susceptibility and the critical slowing down of spin are absent around the end-point, where the divergence of 


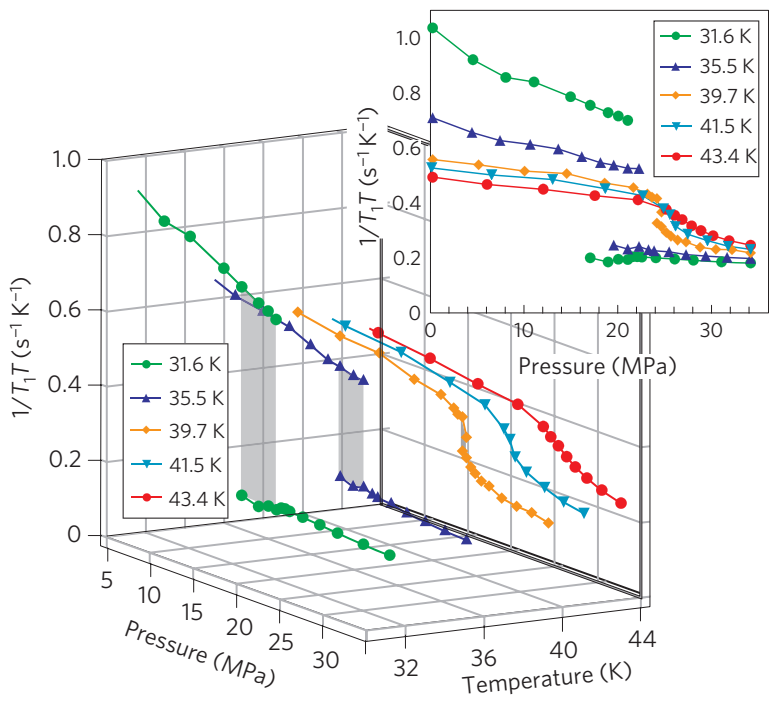

Figure 5 | NMR characteristics around the Mott critical end-point. The pressure dependence of $1 / T_{1} T$ of the outer site at various temperatures. The shaded area represents the coexistence of insulating and bad metallic phases. Inset: The same data as in the main panel shown in a different manner for clarity.

doublon susceptibility and the critical slowing down of doublon density fluctuations are both expected ${ }^{18,19}$. This is a natural consequence of no magnetic symmetry breaking at the end-point.

Since the publication of the Brinkman-Rice approach ${ }^{26}$, the metal-to-insulator Mott transition has often been discussed in terms of the enhancement of single-quasiparticle mass $m^{*}$. In fact, at low temperatures the $m^{*}$ enhancement (not divergent because of the discontinuous Mott transition) was predicted by cluster DMFT (ref. 17) and observed by transport ${ }^{13}$ and optical conductivity ${ }^{23,27,28}$ $\sigma(\omega)$ measurements on $\kappa$-(BEDT-TTF $)_{2}$ X. It should be noted that, because $m^{*}$ is well defined only in the Fermi-liquid regime at low temperatures, $m^{*}$ is probably not an adequate physical parameter to feature the Mott criticality at the end-point situated in the bad metallic regime. The question arising from the present results is why BCMC_-essentially a criticality of charge - is emergent in the spin degree of freedom. Theoretically, the Mott insulator-to-metal transition is captured by the increase in the density of doublons and holons (empty site) $)^{18,21}$, which are 'charge carriers' contributing to the transport properties. In the NMR properties, on the other hand, doublons and holons are probably equivalent to 'spin vacancies', which suppress $1 / T_{1}$. The possibly identical criticality in the transport and magnetism seems to suggest that the magnetic Mott criticality is also dominated by the doublons and holons as a result of their dual nature, that is, charge carrier and spin vacancy.

\section{Methods}

The crystals were synthesized from the BEDT-TTF molecule with two central carbon atoms labelled with ${ }^{13} \mathrm{C}$ isotopes (Fig. 1a) for NMR, which was carried out under 7.4 T $(\| a)$. In this field configuration, all BEDT-TTF molecules are equivalent in the sense of NMR and the dipolar coupling between the adjacent ${ }^{13} \mathrm{C}$ nuclear spins vanishes ${ }^{29}$. Thus, the ${ }^{13} \mathrm{C}$-NMR spectra consist of two lines ${ }^{29}$ coming from the 'inner' and 'outer' sites in BEDT-TTF (see Supplementary Fig. S3). We measured ${ }^{13} \mathrm{C}-\mathrm{NMR}$ spectra and nuclear spin-lattice relaxation rate divided by temperature, $1 / T_{1} T$, around the end-point. $1 / T_{1} T$ depends on the dynamical spin susceptibility, as follows:

$$
1 / T_{1} T \propto \sum_{q} \frac{\operatorname{Im} \chi\left(q, \omega_{0}\right)}{\omega_{0}}
$$

where $\mathbf{q}$ is the wavenumber vector and $\omega_{0}$ is the NMR observation frequency. $1 / T_{1} T$ in $\kappa$-Cl under the present $P-T$ range $(0-35 \mathrm{MPa}$ and $31-43 \mathrm{~K})$ is dominated by the antiferromagnetic fluctuations (that is, $\chi(\mathbf{Q})$, where $\mathbf{Q}$ is the wave vector of antiferromagnetic order) even in the bad metallic phase ${ }^{9}$. Therefore, the temperature or pressure variation of $1 / T_{1} T$ can be interpreted as the variation of antiferromagnetic fluctuations.

The $1 / T_{1} T$ data of the inner and outer sites probe an identical quantity through different proportional constants (so-called hyperfine coupling constants). As the coupling constant is larger at the outer site, we present only the data of the outer sites. Concerning the ${ }^{13} \mathrm{C}-\mathrm{NMR}$ shift, our previous study showed that the shift of $\kappa$-Cl reflects not only the uniform susceptibility but also the staggered susceptibility because of the Dzyaloshinsky-Moriya interaction ${ }^{30}$. Therefore, the interpretation of shift is not straightforward. For this reason, the pressure and temperature dependence of the shift is presented in Supplementary Fig. S2.

\section{Received 1 March 2009; accepted 8 September 2009;} published online 18 October 2009

\section{References}

1. Mott, N. F. Metal-Insulator Transitions (Taylor \& Francis, 1990).

2. Timusk, T. \& Statt, B. The pseudogap in high-temperature superconductors: An experimental survey. Rep. Prog. Phys. 62, 61-122 (1999).

3. Nam, M.-S., Ardavan, A., Blundell, S. J. \& Schlueter, J. A. Fluctuating superconductivity in organic molecular metals close to the Mott transition. Nature 449, 584-587 (2007).

4. Limelette, P. et al. Universality and critical behavior at the Mott transition. Science 302, 89-92 (2003).

5. Kagawa, F., Miyagawa, K. \& Kanoda, K. Unconventional critical behaviour in a quasi-two-dimensional organic conductor. Nature 436, 534-537 (2005).

6. Founier, D., Poirier, M., Castonguay, M. \& Truong, K. Mott transition, compressibility divergence, and the $P-T$ phase diagram of layered organic superconductors: An ultrasonic investigation. Phys. Rev. Lett. 90, 127002 (2003).

7. Souza, de M. et al. Anomalous lattice response at the Mott transition in a quasi-2D organic conductor. Phys. Rev. Lett. 99, 037003 (2007).

8. Millis, A. J., Monien, H. \& Pines, D. Phenomenological model of nuclear relaxation in the normal state of $\mathrm{YBa}_{2} \mathrm{Cu}_{3} \mathrm{O}_{7}$. Phys. Rev. B 42, 167-178 (1990).

9. Yusuf, E., Powell, B. J. \& McKenzie, R. H. Antiferromagnetic spin fluctuations in the metallic phase of quasi-two-dimensional organic superconductors. Phys. Rev. B 75, 214515 (2007).

10. Kanoda, K. Recent progress in NMR studies on organic conductors. Hyperfine Interact. 104, 235-249 (1997).

11. Kino, H. \& Fukuyama, H. Phase diagram of two-dimensional organic conductors: (BEDT-TTF) ${ }_{2}$ X. J. Phys. Soc. Jpn 65, 2158-2169 (1996).

12. Lefebvre, S. et al. Mott transition, antiferromagnetism, and unconventional superconductivity in layered organic superconductors. Phys. Rev. Lett. 85, 5420-5423 (2000).

13. Limelette, P. et al. Mott transition and transport crossovers in the organic compound $\kappa$-(BEDT-TTF $)_{2} \mathrm{Cu}\left[\mathrm{N}(\mathrm{CN})_{2}\right] \mathrm{Cl}$. Phys. Rev. Lett. 91, 016401 (2003).

14. Kagawa, F., Itou, T., Miyagawa, K. \& Kanoda, K. Transport criticality of the first-order Mott transition in the quasi-two-dimensional organic conductor $\kappa$-(BEDT-TTF $)_{2} \mathrm{Cu}\left[\mathrm{N}(\mathrm{CN})_{2}\right] \mathrm{Cl}$. Phys. Rev. B 69, 064511 (2004).

15. Kagawa, F., Itou, T., Miyagawa, K. \& Kanoda, K. Transport criticality of the first-order Mott transition in the quasi-two-dimensional organic conductor $\kappa$-(BEDT-TTF $)_{2} \mathrm{Cu}\left[\mathrm{N}(\mathrm{CN})_{2}\right] \mathrm{Cl}$. Phys. Rev. Lett. 93, 127001 (2004).

16. Ohashi, T., Momoi, T., Tsunetsugu, H. \& Kawakami, N. Finite temperature Mott transition in Hubbard model on anisotropic triangular lattice. Phys. Rev. Lett. 100, 076402 (2008).

17. Park, H., Haule, K. \& Kotliar, G. Cluster dynamical mean field theory of the Mott transition. Phys. Rev. Lett. 101, 186403 (2008).

18. Kotliar, G., Lange, E. \& Rozenberg, M. J. Landau theory of the finite temperature Mott transition. Phys. Rev. Lett. 84, 5180-5183 (2000).

19. Imada, M. Universality classes of metal-insulator transitions in strongly correlated electron systems and mechanism of high-temperature superconductivity. Phys. Rev. B 72, 075113 (2005).

20. Georges, A., Kotliar, G., Krauth, W. \& Rozenberg, M. J. Dynamical mean-field theory of strongly correlated fermion systems and the limit of infinite dimensions. Rev. Mod. Phys. 68, 13-125 (1996).

21. Kotliar, G. \& Vollhardt, D. Strongly correlated materials: Insights from dynamical mean-field theory. Phys. Today 57, 53-59 (2004).

22. Sasaki, T., Yoneyama, N., Kobayashi, N., Ikemoto, Y. \& Kimura, H. Imaging phase separation near the Mott boundary of the correlated organic superconductors $\kappa$-(BEDT-TTF) ${ }_{2}$ X. Phys. Rev. Lett. 92, 227001 (2004).

23. Dumm, M. et al. Bandwidth-controlled Mott transition in $\kappa$-(BEDT-TTF $)_{2} \mathrm{Cu}\left[\mathrm{N}(\mathrm{CN})_{2}\right] \mathrm{Br}_{x} \mathrm{Cl}_{1-x}$ : Optical studies of correlated carriers. Phys. Rev. B 79, 195106 (2009).

24. Kobashi, K. Transport Properties Near the Mott Transition in the Quasi-Two-Dimensional Organic Conductor $\kappa-(E T)_{2} X$. Thesis, Univ. Tokyo (2007).

25. Papanikolaou, S. et al. Universality of liquid-gas Mott transitions at finite temperatures. Phys. Rev. Lett. 100, 026408 (2008).

26. Brinkman, W. F. \& Rice, T. M. Application of Gutzwiller's variational method to the metal-insulator transition. Phys. Rev. B 2, 4302-4304 (1970). 
27. Faltermeier, D. et al. Bandwidth-controlled Mott transition in $\kappa$-(BEDT-TTF $)_{2} \mathrm{Cu}\left[\mathrm{N}(\mathrm{CN})_{2}\right] \mathrm{Br}_{x} \mathrm{Cl}_{1-x}$ : Optical studies of localized charge excitations. Phys. Rev. B 76, 165113 (2007).

28. Merino, J., Dumm, M., Drichko, N., Dressel, M. \& McKenzie, R. H. Quasiparticles at the verge of localization near the Mott metal-insulator transition in a two-dimensional material. Phys. Rev. Lett. 100, 086404 (2008).

29. De Soto, S. M. et al. ${ }^{13} \mathrm{C}$ NMR studies of the normal and superconducting states of the organic superconductor $\kappa-(\mathrm{ET})_{2} \mathrm{Cu}\left[\mathrm{N}(\mathrm{CN})_{2}\right] \mathrm{Br}$. Phys. Rev. B 52, 10364-10368 (1995).

30. Kagawa, F., Kurosaki, Y., Miyagawa, K. \& Kanoda, K. Field-induced staggered magnetic moment in the quasi-two-dimensional organic Mott insulator $\kappa$-(BEDT-TTF $)_{2} \mathrm{Cu}\left[\mathrm{N}(\mathrm{CN})_{2}\right] \mathrm{Cl}$. Phys. Rev. B 78, 184402 (2008).

\section{Acknowledgements}

We thank S. Watanabe, M. Imada, J. Fujioka and Y. Takahashi for fruitful discussion.

This work was partially supported by Grant-in-Aids for Scientific Researches on the
Priority Area (Grant No. 17071003) and on Innovative Area (Grant No. 20110002) from the MEXT, a Grant-in-Aid for Scientific Research (Grant Nos 20244055 and No. 20540346) from the JSPS and the Global COE Program: Global Center of Excellence for the Physical Sciences Frontier.

\section{Author contributions}

F.K., K.M. and K.K. designed the experiments. F.K. carried out the experiments and analysed the data. F.K. and K.K. interpreted the data. K.M. grew the single crystals for the study. F.K. wrote the paper with assistance from K.K. and K.M.

\section{Additional information}

Supplementary information accompanies this paper on www.nature.com/naturephysics. Reprints and permissions information is available online at http://npg.nature.com/ reprintsandpermissions. Correspondence and requests for materials should be addressed to F.K. 